\title{
INTEGRALS OF GROUPS
}

BY

\section{Jo ão Araújo*, Peter J. Cameron**, Carlo Casolo ${ }^{\dagger}$, and Francesco MATUCCI ${ }^{\dagger \dagger}$}

\begin{abstract}
An integral of a group $G$ is a group $H$ whose derived group (commutator subgroup) is isomorphic to $G$. This paper discusses integrals of groups, and in particular questions about which groups have integrals and how big or small those integrals can be. Our main results are:

- If a finite group has an integral, then it has a finite integral.

- A precise characterization of the set of natural numbers $n$ for which every group of order $n$ is integrable: these are the cubefree numbers $n$ which do not have prime divisors $p$ and $q$ with $q \mid p-1$.

- An abelian group of order $n$ has an integral of order at most $n^{1+o(1)}$, but may fail to have an integral of order bounded by $c n$ for constant c.

- A finite group can be integrated $n$ times (in the class of finite groups) for every $n$ if and only if it is a central product of an abelian group and a perfect group.
\end{abstract}

There are many other results on such topics as centreless groups, groups with composition length 2 , and infinite groups. We also include a number of open problems.

* Universidade Aberta, R. Escola Politecnica 147, 1269-001, Lisboa, Portugal and CEMATCiências, Faculdade de Ciências, Universidade de Lisboa, 1749-016, Portugal, joao. araujo@uab.pt

** School of Mathematics and Statistics, University of St. Andrews, UK and CEMATCiências, Faculdade de Ciências, Universidade de Lisboa, 1749-016, Portugal, pjc20@ st-andrews.ac.uk

$\dagger$ Dipartimento di Matematica e Informatica "U. Dini", Università di Firenze, Italia, carlo. casolo@unifi.it

†† Instituto de Matemática, Estatística e Computação Científica, Universidade Estadual de Campinas (UNICAMP), São Paulo, Brasil, francesco@ime.unicamp.br 


\section{Introduction}

Let $G$ be a group. An integral (in the sense of antiderivative) of $G$ is any group $H$ such that $H^{\prime}=G$. Several authors refer to groups with an integral as $C$-groups or commutator-realizable groups.

Questions about integrals of groups were first raised by Bernhard Neumann in [11]. It is surprising how little progress has been achieved on this topic since Neumann's paper. Perhaps the explanation is that the proofs rely as much on intricate constructions as on long arguments (though the proofs of our Theorems 4.4 and 7.1 are fairly substantial).

Some groups have no integral, as observed in Group Properties [4]:

It is not true that every group can be realized as the derived subgroup of another group - for instance, the "characteristically metacyclic and commutator-realizable implies abelian" statement tells us that a group whose first two abelianizations are cyclic, but whose second derived subgroup is not trivial, cannot arise as a derived subgroup.

Indeed, there is no simple test for integrability.

Every abelian group is integrable; so the set of orders of non-integrable groups is a subset of the set of orders of non-abelian groups. A major result, which occupies Section 7, is an exact description of this set, more conveniently expressed in terms of its complement: we show that every group of order $n$ is integrable if and only if $n$ is cube-free and does not have prime divisors $p$ and $q$ such that $q \mid p-1$. This implies, in particular, that for every even integer greater than 4 there is a non-integrable group of order $n$.

We will see in Section 2 that if a finite group has an integral, then it has a finite integral; so it makes sense to ask for a good upper bound for the smallest integral of a finite integrable group. In order to have a good computational test for integrability, it would be useful to have such a bound. We conjecture that an integrable group of order $n$ has an integral of order at most $n^{3}$; this is best possible, as shown by the cyclic group of order 2, whose smallest integrals are the dihedral and quaternion groups of order 8 . We have been unable to find a closed formula for the smallest integral of an abelian group, but we give a number of constructions in Section 4 for small integrals of abelian groups. From these constructions it follows that an abelian group of order $n$ has an integral of order $n^{1+o(1)}$. In the other direction, we show that integrals of abelian groups 
of order $n$ do not have order bounded by $c n$ for any constant $c$. We also give a weaker bound $n^{c \log n}$ for the order of the smallest integral of a centreless group of order $n$.

Also, there are infinite abelian groups $A$ which do not have an integral $G$ with $|G: A|$ finite.

Any perfect group, and in particular any non-abelian simple group, is its own integral. Motivated by this, we analyse non-perfect groups with composition length 2, and decide whether or not such groups are integrable (up to a specific question about outer automorphism groups of simple groups). This question leads to a more general topic: When does a group $G$ have an integral inside some "universal" group $U$ containing $G$ ?

By analogy with $C^{\infty}$ functions in analysis, we are led to the question: does there exist a group which can be integrated infinitely often? Clearly any perfect group has this property, since it is its own integral. Bernhard Neumann [11] showed that there is no such sequence where all the groups are finite and the sequence increases strictly; but we give in Subsection 8.3 two sequences satisfying slightly weaker conditions. We also examine finite groups which can be integrated $n$ times for every positive integer $n$. These turn out to be central products of an abelian group and a perfect group.

We hope that the results about integrals will inspire the research on similar inverse problems in group theory, some of which will be discussed in a following paper.

We are grateful to Alireza Abdollahi, Lars Jaffke, Michael Kinyon, and Avinoam Mann for valuable comments. In particular, Alireza Abdollahi informed us of his paper [1]; although we have not seen the paper, the author kindly communicated to us the main results. He also drew our attention to a comment he had made on MathOverflow [8], and to the paper by Filom and Miraftab [2]. This paper was published in 2017, but the work of these authors was completely independent of ours. Finally, Abdollahi is (as far as we know) the first author to use the term "integral" in the sense used here.

\section{Preliminaries}

In this section we gather some straightforward observations and prove that integrable finite groups have finite integrals. First we note that, if a group has an integral, then it has infinitely many: 
Lemma 2.1: Let $G$ be a group, let $H$ be an integral for $G$ and let $A$ be an abelian group. Then $H \times A$ is an integral for $G$.

This is self-evident and already known (but we do not know where it was first observed). It is the analogue of adding a "constant of integration".

Theorem 2.2: Let $G$ be a finite group. If $G$ has an integral, then it has an integral which is a finite group.

Proof. Let $H$ be an integral of $G$. First, we reduce to the case where $H$ is finitely generated.

Since $G=H^{\prime}$, there are finitely many commutators $\left[h_{1}, k_{1}\right], \ldots,\left[h_{r}, k_{r}\right]$ which generate $G$, with $h_{i}, k_{i} \in H$. Now it is clear that the subgroup of $H$ generated by $h_{1}, \ldots, h_{r}, k_{1}, \ldots, k_{r}$ is an integral of $G$, and is finitely generated. So, without loss, $H$ is finitely generated.

Any conjugacy class in $H$ is contained in a coset of $H^{\prime}=G$. (For $h, x \in H$, we have $G x^{-1} h x h^{-1}=G$, so $G x^{-1} h x=G h$.) Thus $H$ is a BFC-group (the conjugacy classes are finite of bounded order). While not every BFC-group has centre of finite index, this is true for finitely generated BFC-groups. For let $H=\left\langle x_{1}, \ldots, x_{k}\right\rangle$. By assumption, $C_{H}\left(x_{i}\right)$ has finite index in $H$ (at most $\left.|G|\right)$; so $Z(H)=\bigcap_{i=1}^{k} C_{H}\left(x_{i}\right)$ has finite index in $H$.

In consequence, $Z(H)$ is finitely generated abelian. So it has the form $A \times B$, where $A$ is finite and $B$ is finitely generated torsion-free. Since $B \leq Z(H)$, it is a normal subgroup of $H$; since it is torsion-free, $B \cap G=1$. Thus $G$ embeds in the finite group $\bar{H}=H / B$, and $(\bar{H})^{\prime}=G$.

REMARK 2.3: In the paper [2], this theorem is proved under stronger hypotheses. In addition, there are results in the paper which can be improved using our theorem. For example, Theorem 20 asserts that, if a non-abelian 2-group $G$ has cyclic centre and automorphism group a 2-group, then any integral of $G$ is infinite; we can now conclude that such a group is not integrable.

It is possible for a group to have infinitely many integrals with no abelian direct factors (see [12]). For example, every extraspecial $p$-group is an integral of the cyclic group of prime order $p$. (A p-group $P$ is special if $P^{\prime}=\Phi(P)=Z(P)$, and is extraspecial if this subgroup is cyclic of prime order.) 
Proposition 2.4: There exists a function $f$ defined on the natural numbers such that, if $G$ is an integrable group of order $n$, then $G$ has an integral of order at most $f(n)$.

Proof. We can take $f(n)$ to be the maximum, over all integrable groups $G$ of order $n$, of the minimum order of an integral of $G$. (We will see at the end of this section that, for every $n$, there is an integrable group of order $n$, so this function is well-defined.)

An algorithm for deciding whether a group $G$ of order $n$ is integrable could run as follows: examine all groups $H$ of order at most $f(n)$ (and divisible by $n$ ); return true if such an $H$ is found with $H^{\prime}=G$, and false otherwise. However, in the absence of a decent bound for $f(n)$, this algorithm is worthless. It would be useful to have a good bound, and we pose the question whether $f(n)<n^{3}$ for all natural numbers $n$. (Note that the smallest integrals of the cyclic group of order 2 are the dihedral and quaternion groups of order 8.)

In Section 5 we will prove a weaker bound for centreless groups.

Finally, we consider abelian groups. Guralnick [5] showed that, if $A$ is an abelian group of order $n$, then the group $A<C_{2}$, of order $2 n^{2}$, is an integral of $A$. In Section 4 we will find much smaller integrals of abelian groups, and pose the question of finding the smallest.

\section{Some examples of integrability}

In this section we give some examples of integrable and non-integrable groups. These are of some interest in their own right, and will also be used in the discussion of orders of non-integrable groups. We also give some results concerning direct products. We begin by recalling known results in the literature:

(a) Abelian groups are integrable [5].

(b) Dihedral groups are non-integrable. (This is stated by Neumann [11] with a reference to Zassenhaus [18], which we have not been able to check; an explicit proof, also showing that quasi-dihedral and generalized quaternion groups are non-integrable, is in [2, Corollary 18].) 
(c) Symmetric groups $S_{n}$ (for $n \geq 3$ ) are non-integrable. (This is folklore. The first reference we found in print is in [11], where it is stated without proof; the earliest proof we found in print is [2, Corollary 15 and Theorem 16].)

(d) Some matrix groups are integrable [10]. Miller deals with all normal subgroups of the general linear groups $\mathrm{GL}_{n}(K)$, the unitary groups $\mathrm{U}_{n}(K)$, and the orthogonal groups $\mathrm{O}_{n}(K)$ for $K$ a field of characteristic different from 2 .

We will recover some of the results above as consequences of our own constructions.

The next result is essentially the same as [2, Theorem 17].

Proposition 3.1: Let $G$ be a group with a characteristic cyclic subgroup $C$ which is not contained in $Z(G)$. Then $G$ has no integral.

Proof. Suppose for a contradiction that $H^{\prime}=G$. Since $C$ is characteristic in $G$, it is normal in $H$, and $H$ (acting by conjugation) induces a group of automorphisms of $C$. The automorphism group of a cyclic group is abelian, and so $G=H^{\prime}$ acts trivially on $C$, and $C \leq Z(G)$, a contradiction.

Corollary 3.2: If $n$ is even and $n>4$, the dihedral group of order $n$ is non-integrable.

Proof. The cyclic subgroup of order $n / 2$ is characteristic (since all elements outside it have order 2) and non-central (since $n>4$ ).

If $n=p q$, where $p$ and $q$ are primes, then a non-abelian group of order $n$ exists if and only if $q \mid p-1$.

Corollary 3.3: Let $p$ and $q$ be primes and $q \mid p-1$. Then the non-abelian group of order $p q$ is centreless and non-integrable.

There are two non-abelian groups of order $p^{3}$. For $p=2$, these are the quaternion group (which has an integral, namely $\mathrm{SL}(2,3)$ ), and the dihedral group (which does not have an integral).

The pattern is similar for odd $p$. We prove the following: 
Theorem 3.4: Of the two non-abelian groups of order $p^{3}$, where $p$ is an odd prime, the group of exponent $p$ has an integral, while the group of exponent $p^{2}$ does not.

Proof. The group of exponent $p$ is isomorphic to the group $G$ of upper unitriangular $3 \times 3$ matrices over the field of order $p$. A short calculation with matrices shows that $G=H^{\prime}$, where $H$ is the group of upper triangular matrices with non-zero elements on the diagonal.

The group of exponent $p^{2}$ has the presentation

$$
G=\left\langle a, b: a^{p^{2}}=b^{p}=1, b^{-1} a b=a^{p+1} .\right\rangle
$$

We first develop some properties of this group. Note that its centre has order $p$ and is generated by $a^{p}$. Let $B=\left\langle a^{p}, b\right\rangle$. Then $B$ is elementary abelian of order $p^{2}$.

We show first that every element outside $B$ has order $p^{2}$. To see this, note that $b^{j} a b^{-j}=a^{-j p+1}$, so

$$
b^{j} a^{i} b^{-j}=\left(b^{j} a b^{-j}\right)^{i}=a^{i(-j p+1)} .
$$

Then we get

$$
\begin{aligned}
\left(a^{i} b^{j}\right)^{p} & =a^{i} \cdot b^{j} a^{i} b^{-j} \cdot b^{2 j} a^{i} b^{-2 j} \cdots b^{(p-1) j} a^{i} b^{-(p-1) j} \\
& =a^{i} \cdot a^{i(-j p+1)} \cdot a^{i(-2 j p+1)} \cdots a^{i(-(p-1) j p+1)} \\
& =a^{p i}
\end{aligned}
$$

since $j(1+2+\cdots+(p-1))$ is divisible by $p$ (since $p$ is odd) and $a^{p^{2}}=1$. Thus, $a^{i} b^{j}$ has order $p$ if and only if $p$ divides $i$, which means that $a^{i} b^{j} \in B$.

Let $\alpha$ be an automorphism of $G$, and suppose that $a^{\alpha}=a^{i} b^{j}$ (where $p$ does not divide $i$ ) and $b^{\alpha}=a^{p k} b^{l}$ (for if $b^{\alpha} \notin B$, then $b^{\alpha}$ would have order $p^{2}$, which is impossible). We must have

$$
b^{-\alpha} a^{\alpha} b^{\alpha}=\left(a^{\alpha}\right)^{p+1} .
$$

Since $a^{k p}$ is central, for the left-hand side we have

$$
\begin{aligned}
b^{-l}\left(a^{i} b^{j}\right) b^{l} & =b^{-l} a^{i} b^{l} b^{j} \\
& =a^{i(l p+1)} b^{j} .
\end{aligned}
$$

On the right we have

$$
\left(a^{i} b^{j}\right)^{p+1}=\left(a^{i} b^{j}\right)^{p} a^{i} b^{j}=a^{i(p+1)} b^{j} .
$$


So we must have $l=1$. There are $p(p-1)$ choices for $i, p$ for $j$, and $p$ for $k$; so $|\operatorname{Aut}(G)|=p^{3}(p-1)$. The inner automorphism group has order $|G / Z(G)|=p^{2}$, and so the outer automorphism group has order $p(p-1)$.

In more detail: Conjugation by $b$ corresponds to $(i, j, k)=(p+1,0,0)$, while conjugation by $a$ corresponds to $(i, j, k)=(1,0,-1)$. So we can represent the outer automorphism group by pairs $(i(\bmod p), j)$. Calculation shows that this group is isomorphic to the 1-dimensional affine group.

Now suppose that $H$ is an integral of $G$. Then $H$ acts on $G$ by conjugation, so there is a homomorphism $\theta: H \rightarrow \operatorname{Aut}(G)$, whose restriction to $G$ maps $G$ to $\operatorname{Inn}(G)$. Write $\bar{H}$ and $\bar{G}$ for the images of $H$ and $G$ under $\theta$. Then $(\bar{H})^{\prime}=\bar{G}$, hence $\bar{H} / \bar{G}$ is abelian, so either its order is $p$ or it divides $p-1$.

If $\bar{H} / \bar{G}$ has order $p$, then $|\bar{H}|=p^{3}$, and so it is not possible that $\left|(\bar{H})^{\prime}\right|=$ $|\bar{G}|=p^{2}$.

Suppose that $m=|\bar{H} / \bar{G}|$ divides $p-1$. Up to conjugation, we may assume that an element $\bar{h}$ of $\bar{H}$ of order $m$ is represented as an outer automorphism of $G$ by a map with $j=0$. This means that the automorphism fixes $b$. Its action on the quotient $G / Z(G)$, regarded as a 2-dimensional vector space, is a diagonal matrix with eigenvalues $\lambda$ and 1 . An eigenspace with eigenvalue $\lambda$ has the property that all its cosets are fixed. This means that all the automorphisms in $\bar{H}$ fix every coset of a subgroup $K$ of $G$ of order $p^{2}$; so the commutator of any two of them belongs to $K$. So the derived group of $H$ is contained in $K$, and cannot be $G$.

For higher powers of a prime, a similar result holds:

Proposition 3.5: Let $p$ be an odd prime and $n>3$. Let

$$
G=\left\langle a, b \mid a^{p^{n-2}}=1, b^{p^{2}}=1, b^{-1} a b=a^{p^{n-3}+1}\right\rangle .
$$

Then $G$ is not integrable.

Proof. We first deal with the case $n=4$, following the arguments in the proof of Theorem 3.4. In this case, the group $G=G_{4}$ has order $p^{4}$; its centre and Frattini subgroup coincide, and $Z(G)=\left\langle a^{p}, b^{p}\right\rangle$, elementary abelian of order $p^{2}$, while its derived subgroup is generated by $a^{p}$ and is cyclic of order $p$. The calculations in the proof of Theorem 3.4 show that $\left(a^{i} b^{j}\right)^{p}=a^{p i} b^{p j}$. So elements outside $Z(G)$ have order greater than $p$. 
Any automorphism must map $a$ to an element whose $p$ th power lies in the derived group, necessarily of the form $a^{i} b^{p j}$, and $b$ to an element not of this form, say $a^{k} b^{l}$ where $p \nmid l$. Now the proof continues almost exactly as in the proof of Theorem 3.4.

For $n>4$, we can complete the proof by induction. We note that the Frattini subgroup of $G$ is generated by $a^{p}$ and $b^{p}$, and is abelian, with structure $C_{p} \times$ $C_{p^{n-3}}$; its Frattini subgroup $M$ is the group generated by $a^{p^{2}}$ (isomorphic to $C_{p^{n-4}}$ ). Thus $M$ is a nontrivial characteristic subgroup of $G$. If $H$ is an integral of $G$, then $M$ is normal in $H$, and $(H / M)^{\prime}=H^{\prime} / M=G / M \cong G_{4}$. But we showed above that $G_{4}$ is not integrable, so this is a contradiction.

Now we consider product constructions, and show the following.

Proposition 3.6: Let $G=G_{1} \times G_{2}$.

(a) If $G_{1}$ and $G_{2}$ are integrable, then so is $G$.

(b) If $G$ is integrable and $\operatorname{gcd}\left(\left|G_{1}\right|,\left|G_{2}\right|\right)=1$, then $G_{1}$ and $G_{2}$ are integrable.

(c) If $G_{1}$ is centreless and $G_{2}$ is abelian, then $G$ is integrable if and only if $G_{1}$ is integrable.

Proof. (a) Suppose that $H_{i}^{\prime}=G_{i}$ for $i=1,2$, and let $H=H_{1} \times H_{2}$. Then

$$
H^{\prime}=H_{1}^{\prime} \times H_{2}^{\prime}=G_{1} \times G_{2}=G \text {. }
$$

(b) Suppose that $H$ is an integral of $G_{1} \times G_{2}$. Then $G_{1}$ is a characteristic subgroup of $G_{1} \times G_{2}$, and hence is normal in $H$, and is contained in $H^{\prime}$. Thus

$$
\left(H / G_{1}\right)^{\prime}=H^{\prime} / G_{1} \cong G_{2},
$$

so $G_{2}$ is integrable; and similarly $G_{1}$ is integrable.

(c) Suppose that $G_{1} \times G_{2}$ is integrable, say $H^{\prime}=G_{1} \times G_{2}$. By assumption, $G_{2}=Z\left(G_{1} \times G_{2}\right)$, so $G_{2}$ is a characteristic subgroup of $G$, and thus is normal in $H$. Then

$$
\left(H / G_{2}\right)^{\prime}=H^{\prime} / G_{2}=\left(G_{1} \times G_{2}\right) / G_{2} \cong G_{1},
$$

so $G_{1}$ is integrable. The converse is clear.

The "centreless" condition in part (c) is essential. For example, $D_{8}$ is not integrable, but $C_{2} \times D_{8}$ has an integral of order 128 . 


\section{Abelian groups}

As we noted earlier, Guralnick observed that finite abelian groups are integrable. Indeed, $A \nmid C_{2}$ is an integral of the abelian group $A$, and has order $2 n^{2}$ if $|A|=n$.

It is possible to construct much smaller integrals of abelian groups in most cases. If $A$ is an abelian group of odd order, then the group

$$
\left.\langle A, t| t^{2}=1, t^{-1} a t=a^{-1} \text { for all } a \in A\right\rangle
$$

is an integral of $A$ of order $2|A|$. Since any finite abelian group is the direct product of a group of odd order and a 2-group, the results of the last section show that it is enough to consider the latter.

Observe the following:

- If $A \cong\left(C_{2^{m}}\right)^{2}=\left\langle a_{1}, a_{2}\right\rangle$, then

$$
\left\langle A, s: s^{3}=1, s^{-1} a_{1} s=a_{2}, s^{-1} a_{2} s=a_{1}^{-1} a_{2}^{-1}\right\rangle
$$

is an integral of $A$ of the form $A \rtimes C_{3}$.

- If $A \cong\left(C_{2^{m}}\right)^{3}$, there is similarly an integral of $A$ of the form $A \rtimes C_{7}$. (This is a little more complicated than the previous: there we used the integer matrix $\left(\begin{array}{cc}0 & 1 \\ -1 & -1\end{array}\right)$ of order 3 . There is no $3 \times 3$ integer matrix of order 7; but there is such a matrix over the 2-adic integers. Equivalently, $\left(C_{2}\right)^{3}$ has an automorphism of order 7 (and is the derived subgroup of the semidirect product), and this automorphism can be lifted to $\left(C_{2^{m}}\right)^{3}$ for all $m$.)

- If $A \cong C_{2^{m}}$, then the dihedral group of order $2^{m+2}$ is an integral of $A$.

Thus, a finite abelian group $A$ has an integral of order at most $42 \cdot 2^{m} \cdot|A|$, where $m$ is the number of powers $2^{a}$ for which the expression for $A$ as a direct product of cyclic groups of prime power order has a unique factor of order $2^{a}$. For this we extend each such cyclic factor to one twice as large; then extend by a cyclic group of order 42 , where the element of order 2 inverts these cyclic groups and the odd-order part of $A$, while elements of orders 3 and 7 act as previously described on products of two or three cyclic 2-groups of the same order. (Any number greater than 1 can be written as a sum of $2 \mathrm{~s}$ and $3 \mathrm{~s}$.) Noting that $|A| \geq 2^{1+2+\cdots+m}=2^{m(m+1) / 2}$, we see that the order of this integral is at most $|A|^{1+o(1)}$. 
Can this bound be reduced to $c|A|$ for some constant $c$ ? We see that, to answer this question, we need to consider direct products of cyclic 2 -groups of distinct orders.

Lemma 4.1: Let $H$ be a 2-group acting by automorphisms on the finite elementary abelian 2-group $A$, then

$$
|A /[A, H]| \geq|A|^{1 /|H|} .
$$

Proof. By induction on $|H|$. Let $H=\langle x\rangle$ have order 2. Then for every $a \in A$,

$$
[a, x]^{x}=[a, x]^{-1}=[a, x],
$$

hence $[A, x] \leq C_{A}(x)$. On the other hand, the map $a \mapsto[a, x]$ is a homomorphism of the abelian group $A$, and so

$$
\left|\frac{A}{[A, x]}\right| \geq\left|\frac{A}{C_{A}(x)}\right|=|[A, x]|
$$

which is what we want.

Let now $|H| \geq 4$, let $Z$ be a central subgroup of order 2 of $A$ fixed by $H$, and $\bar{A}=A /[A, Z]$. Then $H / Z$ acts on $\bar{A}$ and, by inductive assumption,

$$
|\bar{A} /[\bar{A}, H]| \geq|\bar{A}|^{1 /|H / Z|}=|\bar{A}|^{2 /|H|} .
$$

Now clearly $[\bar{A}, H]=[A, H] /[A, Z]$, whence

$$
\left|\frac{A}{[A, H]}\right|=\left|\frac{\bar{A}}{[\bar{A}, H]}\right| \geq|\bar{A}|^{2 /|H|} \geq\left(|A|^{1 / 2}\right)^{2 /|H|}=|A|^{1 /|H|} .
$$

Lemma 4.2: Let $A$ be a finite elementary abelian 2-group, and $G$ a 2-group such that $G^{\prime}=A$; writing $H=G / A$, we have

$$
|H| \log ^{2}|H| \geq 2 \log |A| .
$$

Proof. Let $T=[A, G]$. Then, by Lemma 4.1,

$$
|A|^{1 /|H|} \leq|A / T|
$$

On the other hand, $A / T=(G / T)^{\prime}$ is an elementary abelian subgroup of $Z(G / T)$; by standard arguments it follows that $G / T$ modulo its centre is elementary abelian of order, say, $2^{t}$; moreover

$$
|A / T| \leq 2^{\left(\begin{array}{c}
t \\
2
\end{array}\right)} \leq|H|^{(t-1) / 2} \leq|H|^{\log |H| / 2} .
$$


Hence

$$
|A|^{1 /|H|} \leq|H|^{\log |H| / 2},
$$

i.e. $|H| \log ^{2}|H| \geq 2 \log |A|$.

Proposition 4.3: Let $A$ be an abelian 2-group which is a direct product of $m$ cyclic groups of distinct orders. Suppose that $A=G^{\prime}$ for some group $G$. Then $|G: A| \rightarrow \infty$ as $m \rightarrow \infty$.

Proof. Let $A=C_{2^{a_{1}}} \times \cdots \times C_{2^{a}}$, with $a_{1}, \ldots, a_{m}$ in strictly decreasing order. The Frattini subgroup $\Phi(A)$ has the property that $A / \Phi(A)$ is elementary abelian of order $2^{m}$, and automorphisms of $A$ of odd order act faithfully on $A / \Phi(A)$. There can be no such non-identity automorphisms. For the subgroups of $A$ consisting of elements of orders dividing $2^{a_{i}}$ are characteristic, and their projections onto $A / \Phi(A)$ form a composition series for this group, whose terms are necessarily fixed by automorphisms of odd order. So elements of odd order in $G$ centralise $A$, and we can assume without loss of generality that $G$ is a 2-group. Now the result follows from Lemma 4.2.

We conclude:

Theorem 4.4: (a) A finite abelian group $A$ has an integral of order at most $|A|^{1+o(1)}$.

(b) There is no constant $c$ such that every finite abelian group $A$ has an integral of order at most $c|A|$.

The arguments above can be refined to give explicit upper and lower bounds for the order of the smallest integral of an abelian group.

We have computed the smallest integrals of abelian 2-groups of orders up to 64. The results are in Table 1. The computations involved simply testing the groups $H$ in the SmallGroups library in GAP to decide whether $H^{\prime}$ is isomorphic to the given group $G$.

\section{Centreless groups}

A group $G$ is complete if its centre is trivial and $\operatorname{Aut}(G)=\operatorname{Inn}(G)$. Equivalently, a group $G$ is complete if and only if, for any group $H$ such that $G \unlhd H$, then $H \cong G \times T$, for some group $T$. This follows from [15, Theorems 7.15 and 7.17] or [14, Theorem 13.5.7]. This has the following consequence: 


\begin{tabular}{|c|c|c|c|c|c|}
\hline Order & $\begin{array}{c}\text { Invariant } \\
\text { factors }\end{array}$ & $\begin{array}{l}\text { Smallest } \\
\text { integral }\end{array}$ & \multirow[b]{3}{*}{ Order } & \multirow{3}{*}{$\begin{array}{c}\text { Invariant } \\
\text { factors }\end{array}$} & \multirow{3}{*}{$\begin{array}{l}\text { Smallest } \\
\text { integral }\end{array}$} \\
\hline 2 & $(2)$ & 8 & & & \\
\hline 4 & $\begin{array}{c}(4) \\
(2,2)\end{array}$ & $\begin{array}{l}16 \\
12\end{array}$ & & & \\
\hline 8 & $\begin{array}{c}(8) \\
(4,2) \\
(2,2,2)\end{array}$ & $\begin{array}{l}32 \\
64 \\
56\end{array}$ & \multirow[t]{3}{*}{64} & $\begin{array}{c}(64) \\
(32,2)\end{array}$ & $\begin{array}{l}256 \\
512\end{array}$ \\
\hline 16 & $\begin{array}{c}(16) \\
(8,2) \\
(4,4) \\
(4,2,2) \\
(2,2,2,2)\end{array}$ & $\begin{array}{c}64 \\
128 \\
48 \\
128 \\
48\end{array}$ & & $\begin{array}{c}(10,4) \\
(16,2,2) \\
(8,8) \\
(8,4,2) \\
(8,2,2,2)\end{array}$ & $\begin{array}{l}512 \\
512 \\
192 \\
512 \\
512\end{array}$ \\
\hline 32 & $\begin{array}{c}(32) \\
(16,2) \\
(8,4) \\
(8,2)\end{array}$ & $\begin{array}{l}128 \\
256 \\
256 \\
256\end{array}$ & & $\begin{array}{c}(4,4,4) \\
(4,4,2,2) \\
(4,2,2,2,2) \\
(2,2,2,2,2,2) \\
\end{array}$ & $\begin{array}{l}440 \\
192 \\
512 \\
192 \\
\end{array}$ \\
\hline & $\begin{array}{c}(4,4,2) \\
(4,2,2,2) \\
(2,2,2,2,2)\end{array}$ & $\begin{array}{l}256 \\
256 \\
256 \\
\end{array}$ & & & \\
\hline
\end{tabular}

Table 1. Smallest integrals of abelian groups

Proposition 5.1: Let $G$ be a complete group. Then $G$ is integrable if and only if it is perfect.

Proof. Suppose that $H^{\prime}=G$. Then $G \unlhd H$, so $H \cong G \times T$ for some $T \unlhd H$ with $G \cap T=1 ;$ and $T \cong G T / G \cong H / H^{\prime}$ is abelian, and so $G^{\prime}=H^{\prime}=G$.

The converse is trivial since every perfect group is integrable.

We now turn to the more general class of centreless groups (those with trivial centre).

If $Z(G)=1$, then $G$ is isomorphic to a subgroup of $\operatorname{Aut}(G)$, namely the group of inner automorphisms of $G$. Furthermore, $\operatorname{Aut}(G)$ also has trivial centre. So the process can be continued:

$$
G \leq \operatorname{Aut}(G) \leq \operatorname{Aut}(\operatorname{Aut}(G)) \leq \cdots .
$$


Wielandt's automorphism tower theorem (for example, see [17, Theorem 13.5.4]) says that the procedure terminates after finitely many steps. The final group in the sequence is complete.

In this section, we show that the same is true for reduced integrals of $G$. Let $G$ be a group with $Z(G)=1$. We say that an integral $H$ of $G$ is reduced if $C_{H}(G)=1$. Asking for a reduced integral removes the "constant of integration" (abelian direct factor), but does more than this.

For example, let $G=A_{5}$, and let $H$ be a semidirect product of $G$ with a cyclic group of order 4 whose generator induces on $G$ the automorphism of conjugation by a transposition. Then $C_{H}(G)$ is cyclic of order 2 , and $H / C_{H}(G)$ is an integral of $G$ isomorphic to $S_{5}$.

Lemma 5.2: If $Z(G)=1$ and $H$ is an integral of $G$, then

(a) $C_{H}(G)=Z(H)$;

(b) $H / C_{H}(G)$ is a reduced integral of $G$.

Proof. (a) Clearly $C_{H}(G) \geq Z(H)$. Take $h \in C_{H}(G)$, so that $g^{h}=g$ for all $g \in G$. Let $t$ be any other element of $H$. Then $g^{t} \in G=H^{\prime} \unlhd H$. Then

$$
g^{[h, t]}=g^{h^{-1} t^{-1} h t}=g
$$

for all $g \in G$, so $[h, t] \in C_{H}(G)$. But $[h, t] \in H^{\prime}=G$, and $G \cap C_{H}(G)=Z(G)=$ 1. So $[h, t]=1$ for all $t \in H$, whence $h \in Z(H)$.

(b) We have

$$
\left(H / C_{H}(G)\right)^{\prime}=H^{\prime} C_{H}(G) / C_{H}(G)=G C_{H}(G) / C_{H}(G) \cong G /\left(G \cap C_{H}(G)\right)=G,
$$

so $H / C_{H}(G)$ is an integral of $G$. But $H / C_{H}(G)$ is isomorphic to the group of automorphisms of $G$ induced by $H$; so $H / C_{H}(G)$ acts faithfully on $G$, whence the centraliser of $G$ is trivial.

Note that, if $Z(G)=1$ and $H$ is a reduced integral of $G$, then $Z(H)=1$, so the process can be continued.

Proposition 5.3: Let $G$ be a finite group with $Z(G)=1$. Suppose that

$$
G=G_{0}<G_{1}<G_{2}<\cdots,
$$

where $G_{n+1}$ is a reduced integral of $G_{n}$ for all $n$. Then the sequence terminates after finitely many steps. 
Proof. Note that $G_{0}$ is normal in $G_{n}$ for all $n$, since it is the $n$th term of the derived series of $G_{n}$.

We prove that $C_{G_{n}}\left(G_{0}\right)=1$ for all $n$. The proof is by induction on $n$; it holds by definition for $n=1$. So let us assume the result for $n$ and take $g \in C_{G_{n+1}}\left(G_{0}\right)$.

For any $h \in G_{n+1}$, we see that $[g, h]$ centralises $G_{0}$, and $[g, h] \in G_{n}$; so $[g, h] \in C_{G_{n}}\left(G_{0}\right)$, whence $[g, h]=1$. As this is true for all $h \in G_{n+1}$, we have $g \in Z\left(G_{n+1}\right)$. But by Lemma 5.2 and the construction, this forces $g=1$.

Now this means that $G_{n}$ is embedded in $\operatorname{Aut}\left(G_{0}\right)$ for all $n$, so $\left|G_{n}\right|$ is bounded by a function of $G_{0}$, and the sequence terminates.

Corollary 5.4: Let $G$ be a group of order $n$ with $Z(G)=1$. Then, if $G$ is integrable, it has an integral of order at most $n^{\log _{2} n}$.

Proof. $G$ can be generated by at $\operatorname{most} \log _{2} n$ elements (using Lagrange's theorem, since by induction on $m$ the group generated by $m$ independent elements has order at least $2^{m}$ ). Now an automorphism is determined by its effect on the generators, and each generator has at most $n$ possible images under any automorphism.

\section{Groups with composition length 2}

In this section, we analyse groups with composition length at most 2 , and give a procedure to determine whether or not such groups are integrable, up to a specific question about outer automorphism groups of simple groups.

First we consider groups with composition length 1, and observe:

Proposition 6.1: If $G$ is a finite simple group, then $G$ has an integral.

Proof. This is easily seen by considering three cases:

- If $G=C_{2}$, then the dihedral and quaternion groups $D_{8}$ and $Q_{8}$ are integrals of $G$.

- If $G=C_{p}$, then the dihedral group of order $2 p$ is an integral of $G$.

- If $G$ is a non-abelian simple group, then it is perfect, so it is its own integral. 
For groups with composition series of length 2, we begin with a simple observation.

Lemma 6.2: Let $G$ be a group with the property that, in every composition series $G>N>\cdots$ for $G$, the factor group $G / N$ is a non-abelian simple group. Then $G$ is perfect, and hence is its own integral.

Proof. For if $G$ is not perfect, then it has an abelian quotient, and hence a normal subgroup with quotient $C_{p}$. Taking this as the start of a composition series gives the result.

Now let $G$ be a group with composition series $G>N>\{1\}$. By Lemma 6.2, we may suppose that $G / N \cong C_{p}$ for some prime $p$.

Case 1: $N \cong C_{q}$ for some prime $q$. There are two possibilities:

- $G=C_{p} \times C_{q}$. Then $G$ is abelian, and so has an integral.

- $p \mid q-1$ and $G$ is non-abelian. Then $G$ does not have an integral. For suppose that $H$ is an integral of $G$. Since $N=G^{\prime}$, we see that $N$ is normal in $H$. The automorphism group of the cyclic group $N$ is abelian, and so $H^{\prime}$ acts trivially on $N$. This contradicts the fact that $H^{\prime}=G$ and $G$ induces a group of order $p$ of automorphisms of $N$.

Case 2: $N$ is a non-abelian simple group. Again there are two subcases:

- If $C_{p}$ induces the trivial outer automorphism of $N$, then we can change the generator by an element of $N$ so that it acts trivially on $N$; then $G=N \times C_{p}$, and both factors have integrals, and hence so does $G$.

- In the other case, $C_{p}$ is a subgroup of $\operatorname{Out}(N)$. If $H$ is an integral of $G$, then $H / N$ is an integral of $G / N \cong C_{p}$ inside $\operatorname{Out}(N)$.

But such a subgroup may or may not exist. (For example, if $\operatorname{Out}(N) \cong$ $D_{6}$ and $p=2$, then $C_{2}$ has an integral, but not within $\operatorname{Out}(N)$, so $G$ has no integral.) Resolving this case will require some detailed analysis of the outer automorphism groups of simple groups.

The previous discussion allows us to recover the following folklore result (see $[10])$.

Proposition 6.3: For every $n \geq 5$, symmetric group $S_{n}$ is non-integrable.

Proof. For every $n \geq 5$, the symmetric group $S_{n}$ has composition length 2 with series $S_{n}>A_{n}>\{1\}$. We recall that $\operatorname{Out}\left(A_{6}\right) \cong C_{2} \times C_{2}$ and $\operatorname{Out}\left(A_{n}\right) \cong C_{2}$ 
for all $n \neq 6$. Since $T:=A_{n} / S_{n} \cong C_{2}$ and its smallest integral $S$ is $D_{8}$, then $S$ is not contained in $\operatorname{Out}\left(A_{n}\right)$ for any $n \geq 5$, the discussion above implies that $S_{n}$ is non-integrable.

\section{Orders of non-integrable groups}

This section is devoted to the proof of the following theorem:

Theorem 7.1: Let $n$ be a positive integer. Then every group of order $n$ is integrable if and only if $n$ is cube-free and there do not exist prime divisors $p$, $q$ of $n$ with $q \mid p-1$.

REMARK 7.2: It is a formal consequence of the statement of this theorem that non-integrable groups of all even orders greater than 4 exist: if $n$ is even and $n>4$ then either $n=2^{d}$ for $d \geq 3$, or $n$ is divisible by an odd prime $p$ (with $2 \mid p-1$ ). However, we already know this because of Corollary 3.2, and we use this result in the proof; so we assume that $n$ is odd.

REMARK 7.3: It is interesting to compare this theorem with the description of numbers $n$ for which all groups of order $n$ are abelian. Since abelian groups are integrable, this set is a subset of the set in the Theorem. It is known that all groups of order $n$ are abelian if and only if $n$ is cube-free and there do not exist primes $p$ and $q$ such that either

- $p$ and $q$ divide $n$, and $q \mid p-1$; or

- $p^{2}$ and $q$ divide $n$, and $q \mid p+1$.

(This result is folklore. For a proof by Robin Chapman, see [7].) So the two sets contain no non-cube-free integers, and coincide on squarefree integers; but there are integers (such as 75) for which non-abelian groups exist but all groups are integrable.

Proof. We show that the conditions on $n$ in the Theorem are necessary. If $n$ is not cube-free, then $n=p^{a} m$ where $p$ is prime, $a \geq 3$, and $p \nmid m$. By Theorem 3.4 and Proposition 3.5, there is a non-integrable group $P$ of order $p^{a} ;$ since $\operatorname{gcd}\left(p^{a}, m\right)=1$, then the direct product of $P$ with any group of order $m$ is not integrable, by Proposition 3.6(b). If primes $p$ and $q$ divide $n$, with $q \mid p-1$, then the non-abelian group of order $p q$ is centreless and non-integrable by Corollary 3.3, so its direct product with any group of order $n / p q$ is nonintegrable, by Proposition 3.6(c). 
So suppose that $n$ satisfies these conditions. If $n$ is even, then $n=2$ or $n=4$; then all groups or order $n$ are abelian, and so integrable (Section 4 ). So we may assume from now on that $n$ is odd. Assume (for a contradiction) that there exists a non-integrable group of order $n$; inductively, we may suppose that $n$ is minimal subject to this.

Our strategy is to show that $G$ has a normal subgroup $N$ which is a direct product of elementary abelian groups of order $p^{2}$ for various primes $p$, and an abelian complement $H$ which normalises each of these factors of $N$, whose action on $N$ gives each factor (of order $p^{2}$, say) the structure of the additive group of $\operatorname{GF}\left(p^{2}\right)$ such that the action of $H$ on $N$ corresponds to multiplication by a subgroup of the multiplicative group of the finite field of order dividing $p+1$.

When this is achieved, we let $K=\langle G, t\rangle$, where $t^{2}=1, t$ normalises $N$ and acts on each factor of order $p^{2}$ as the field automorphism of order 2 of $\operatorname{GF}\left(p^{2}\right)$ (that is, as the map $x \mapsto x^{p}$ ), and on $H$ by inversion (the map $x \mapsto x^{-1}$ ). A short calculation shows that this gives an action of $t$ by automorphism on $G$. (If the order of an element $x$ of the multiplicative group of $\operatorname{GF}\left(p^{2}\right)$ divides $p+1$, then $x^{p}=x^{-1}$.) Now commutators $[t, g]$ for $g \in G$ generate $G$. (If $h \in H$, then $[h, g]=h^{-2}$; since $|H|$ is odd, these elements generate $H$. If $P$ is a Sylow $p$-subgroup of $N$, then the commutators $[g, t]$, for $g \in P$, generate a non-trivial subgroup of $P$; since $H$ acts irreducibly on $P$, the $H$-conjugates of this generate $P$.) So $K^{\prime}=G$, contradicting the assumption that $G$ is not integrable.

So it remains to prove that $G$ has the structure described above. In particular, we have to show that $G$ is metabelian. The Odd-Order Theorem shows that $G$ is soluble; but this can be proved much more easily using Burnside's transfer theorem, as follows.

Since $n$ is cube-free, the Sylow $p$-subgroups of $G$ have order $p$ or $p^{2}$, and so are abelian. Let $p_{1}<p_{2}<\ldots<p_{r}$ be the primes dividing $n$. Let $P_{1}$ be a Sylow $p_{1}$-subgroup of $G$. Now the automorphism group of $P_{1}$ has order $p_{1}-1$, $p_{1}\left(p_{1}-1\right)$ or $p_{1}\left(p_{1}-1\right)^{2}\left(p_{1}+1\right)$, the fact that $p_{1}$ is the smallest prime divisor of $n$ and is odd shows that $N_{G}\left(P_{1}\right)$ acts trivially on $P_{1}$, and so is equal to $C_{G}\left(P_{1}\right)$. By Burnside's Transfer Theorem, $G$ has a normal $p_{1}$-complement $G_{1}$. By induction we construct normal subgroups $G_{2}, \ldots, G_{r}=1$ so that $G_{i+1}$ is a normal $p_{i}$-complement in $G_{i}$, and the quotient is abelian. Thus $G$ is soluble, as claimed. 
Let $F$ be the Fitting subgroup of $G$, the largest nilpotent normal subgroup of $G$. Since $F$ is the direct product of its Sylow subgroups, and these are abelian, $F$ is abelian. Moreover, $F$ contains its centraliser [3, Theorem 6.1.3], and so $C_{G}(F)=F$. Thus, $G / F$ is isomorphic to the group of automorphisms of $F$ induced by conjugation in $G$. This group is a subdirect product of the groups induced on the Sylow subgroups of $F$.

Now the automorphism group of $C_{p}$ is $C_{p-1}$; the automorphism group of $C_{p^{2}}$ is $C_{p(p-1)}$; and the automorphism group of $C_{p} \times C_{p}$ is $\mathrm{GL}(2, p)$, of order $p(p-1)^{2}(p+1)$. Now if $p$ divides $n$, then $p-1$ is coprime to $n$ by assumption; and if $F$ contains a Sylow $p$-subgroup then $p$ does not divide $|G / F|$. Thus the group induced on a Sylow $p$-subgroup $P$ of $F$ is trivial if $P$ is cyclic, and has order dividing $p+1$ if $P$ is elementary abelian of order $p^{2}$. Moreover, from the structure of $\operatorname{GL}(2, p)$, we see that a subgroup of order dividing $p+1$ is cyclic, and corresponds to multiplication by an element in the multiplicative group of $\mathrm{GF}\left(p^{2}\right)$ acting on the additive group of this field.

So $G / F$ is a subdirect product of cyclic groups, and hence is abelian. Moreover, cyclic Sylow subgroups of $F$ are central in $G$.

Now we define $N$ to be the product of the Sylow subgroups of $F$ which are elementary abelian of prime squared order. We see that $G / N$ has a subgroup $F / N$ (generated modulo $N$ by the cyclic Sylow subgroups of $F$ ) with $(G / N) /(F / N) \cong G / F$ abelian. Thus $G / N$ is an extension of a central subgroup by an abelian group, and so it is nilpotent of class at most 2 . But then it is a direct product of its Sylow subgroups, and so is abelian.

We also note that, if $G / N$ acts trivially on a Sylow $p$-subgroup $P$ of $N$, then by Burnside's transfer theorem, $G \cong P \times G_{1}$ for a subgroup $G_{1}$ of order $n / p^{2}$; by the minimality of $n$, we have that $G_{1}$ is integrable, and has order coprime to $p$, so that $G$ is integrable, a contradiction. So the action of $G / N$ on each Sylow subgroup is non-trivial, and each such subgroup has the structure of a finite field $\operatorname{GF}\left(p^{2}\right)$, with the induced automorphism group isomorphic to a subgroup of the multiplicative group, acting irreducibly.

Finally, $N$ is a normal Hall subgroup of $G$; if we take $H$ to be a Hall subgroup for the complementary set of primes, then $H$ is a complement for $N$ in $G$, and $H \cong G / N$, so $H$ is abelian, and we have reached our goal.

\section{Miscellanea}


8.1. Products, subgroups, quotients. We saw in Proposition 3.6(a) that, if $G_{1}$ and $G_{2}$ have integrals, then so does $G_{1} \times G_{2}$. But what about the converse? In other words, is it possible that $G_{1} \times G_{2}$ has an integral but $G_{1}$ and $G_{2}$ do not? We saw earlier in Proposition 3.6(b) that this is not possible if the orders of $G_{1}$ and $G_{2}$ are coprime.

A particular case of the above question is: can $G \times G$ be integrable when $G$ is not integrable? We do not have an example. The smallest non-integrable group is $S_{3}$; and $S_{3} \times S_{3}$ is also non-integrable. (For this group has trivial centre, so if it were integrable it would have a reduced integral, which would be contained in the automorphism group of $S_{3} \times S_{3}$; but this automorphism group has order 72 , and its derived group has order 18.)

The next case is $D_{8} \times D_{8}$. We have shown that it has no integral of order at most 512 .

A related question would replace direct product by central product. However, this question has a negative answer. It is well-known that $D_{8} \circ D_{8}$ is isomorphic to $Q_{8} \circ Q_{8}$, which has the integral $\mathrm{SL}(2,3) \circ \mathrm{SL}(2,3)$.

Regarding semidirect products, there are groups $H$ and $G$, with $H$ acting on $G$ into two different ways, say $\phi$ and $\psi$, such that the semidirect product induced by $\phi$ is integrable but the semidirect product induced by $\psi$ is not. For one such example take $G=C_{4}$ and $H=C_{2}$, and the two possible actions of $H$ on $G$ (the trivial action, and the action by inversion). There are also groups $G$ and $H$ such that every semidirect product they can form fails to be integrable: take the dihedral group of order 10 and the Klein four group. Finally, all semidirect products of two copies of $C_{2}$ are integrable. So all possibilities involving integrals of semidirect products can occur.

Regarding subgroups and homomorphic images, note that the group $G=A_{5}$ (which is perfect, so integrable) contains $H$, the dihedral group of order 10 , which is not integrable (by Proposition 5.1), but has a normal subgroup $K$, the cyclic group of order 5 , such that $H$ is the normalizer of $K$ and both $K$ and $H / K$ are integrable. So neither integrability nor non-integrability is subgroupclosed, and a group can have the property that all its non-trivial proper normal subgroups are integrable with integrable quotient without itself being integrable. Moreover, any finite group has both integrable and non-integrable overgroups (since the alternating group is integrable but the symmetric group is usually not). 
In the reverse direction, we have the following:

Proposition 8.1: Let $G$ be an integrable finite group. Then either $G$ is simple, or $G$ has a non-trivial proper quotient which is integrable.

Proof. Let $H$ be an integral of $G$. There are two cases:

Case 1: $G$ has a non-trivial proper characteristic subgroup $N$ (one invariant under all automorphisms of $G$ ). Then $N$ is normal in $H$, and we have $(H / N)^{\prime}=H^{\prime} / N=G / N$.

Case 2: $G$ is characteristically simple. In this case, $G$ is a direct product of isomorphic simple groups. So either $G$ is simple, or it has a simple (and hence integrable) proper quotient.

8.2. Relative integrals. Let $U$ be a "universal" group. Can we decide, for members $G$ of some class of subgroups of $U$, whether or not $G$ has an integral within $U$ ?

This question includes several special cases which have arisen elsewhere in this paper:

- $U=\operatorname{Aut}(T)$ for some non-abelian simple group $T$, and $G$ is a subgroup containing $T$.

- $U$ is the affine group $\operatorname{AGL}(d, p)$, and $G$ is a subgroup containing the translation group.

- $U$ is the symmetric group $S_{n}$, and $G$ is a (transitive, or maybe 2homogeneous) permutation group of degree $n$.

The third problem for 2-transitive groups involves solving some special cases of the other two, since a 2-transitive group is either affine or almost simple.

More generally, we could ask for a classification of the primitive groups $H \leq$ $S_{n}$ that have a given 2-homogeneous group $G \leq S_{n}$ in their integrals tower. (Equivalently, find the primitive subgroups of $S_{n}$ that appear as the derived group of the derived group of the ... of a given 2-homogeneous group $G \leq$ $S_{n}$.) This question should not be difficult; examining the known list of 2homogeneous groups should give a solution, since in all cases the derived length of the soluble residual is very small. 
8.3. Infinitely integrable GRoups. There are groups which can be integrated $n$ times (that is, which are isomorphic to the $n$th derived group of another group), for all $n$. For example, additive groups of rings with identity have this property: if $G$ is the group of upper unitriangular $\left(2^{n}+1\right) \times\left(2^{n}+1\right)$ matrices over the ring $R$ with identity, then the $n$th derived group of $G$ consists of unitriangular matrices with zeros in all above-diagonal positions except the top right, and is isomorphic to the additive group $R^{+}$of $R$. (We note that, since an upper unitriangular matrix has determinant 1, Cramer's rule shows that its inverse can be found by ring operations alone, and so any product of commutators in this group over any ring with identity can be computed with ring operations.)

We have the following result about groups with this property.

Theorem 8.2: (a) Let $G$ be a finite abelian group; then $G$ can be integrated $n$ times for every natural number $n$ (even within the class of finite nilpotent groups).

(b) A finite group can be integrated $n$ times for every natural number $n$ if and only if it is central product of a perfect group and an abelian group.

Proof. (a) Since any finite abelian group is a direct product of finite cyclic groups, it is enough to show the result for these. Now the cyclic group $C_{n}$ is the additive group of the ring $R_{n}=\mathbb{Z} / n \mathbb{Z}$, and so is the $n$th derived group of the group of upper unitriangular matrices of order $2^{n}+1$ over $R_{n}$. Since the group of upper unitriangular matrices is nilpotent, the result is proved.

Observe that, in this way, one may realize any finite abelian group $A$ as the $n$th derived group of a nilpotent group, in which $A$ is central.

(b) Let $G=N A$, with $N, A$ normal subgroups, $N$ perfect, $A$ abelian, and $A \cap N \leq Z(N)$, and let $n$ be a natural number. By point (a) there exists a group $R$ such that $A=R^{(n)}$ and $A \leq Z(R)$. Now, if $H$ is the central product $H=N \circ R$, amalgamating $A \cap N$ as in $G$, then $H^{(n)}=G$.

Conversely, let $G$ be a finite group. Then there is $k$ such that $X^{(k)}$ is perfect for every $X \leq \operatorname{Aut}(G)$. Let $N$ be the soluble residual of $G$ (the smallest normal subgroup such that $G / N$ is soluble); then $N$ is characteristic in $G$. Suppose there exists a group $H$ such that $G=H^{(n)}$, for some $n \geq k$, and let $C=C_{H}(G)$. Then $N C / C$ is the soluble residual of $H / C$ and $N C \geq H^{(k)} \geq G$. Hence, 
$A=G \cap C \leq Z(G)$ and $G=N C \cap G=N(C \cap G)=N A$, thus proving the claim.

Combining this result with Theorem 7.1, we obtain the following result:

Corollary 8.3: For a natural number $n$, the following are equivalent:

(a) every group of order $n$ is abelian;

(b) every group of order $n$ can be integrated twice;

(c) every group of order $n$ can be integrated $k$ times, for every natural number $k$;

(d) $n$ is cubefree and has no prime divisors $p$ and $q$ such that either $q \mid p-1$, or $q \mid p+1$ and $p^{2} \mid n$.

Proof. We saw in Section 7 the (classical) equivalence of (a) and (d). We have observed that (a) implies (c), and trivially (c) implies (b). So suppose that $n$ is such that every group of order $n$ can be integrated twice. Then by Theorem 7.1, $n$ is cubefree and has no prime divisors $p$ and $q$ with $q \mid p-1$. So suppose that $p$ and $q$ are primes, with $q \mid p+1$ and $p^{2} \mid n$. Let $H$ be the group of order $p^{2} q$ which is a semidirect product of the additive group $N$ of the field of order $p^{2}$ with a subgroup of index $q$ in the multiplicative group. Then as argued there, the only reduced integral of $G$ is obtained by adjoining the Frobenius automorphism $t$ of the field. If $\langle G, t\rangle$ is integrable, then so is $\langle G, t\rangle / N$. But this group is dihedral of order $2 q$, with $q$ odd, and so is not integrable.

One can ask if there are infinitely integrable groups. More precisely, even if a group $G$ is not solvable, one can ask whether there exists an infinite chain of finite groups of the form

$$
G=G_{1}^{\prime} \leq G_{1}=G_{2}^{\prime} \leq G_{2}=G_{3}^{\prime} \leq \ldots
$$

The answer to this question is positive if we allow perfect groups, by taking $G_{i}=G$ for all $i$. However, Neumann [11, Corollary 7.5] showed that there is no strictly ascending infinite sequence if $G_{2}$ is finitely generated; so in particular, there is no such sequence of finite groups. (Note that Neumann [11] gives an example of an infinite ascending sequence where $G_{0}$ and $G_{1}$ are finite and the other terms infinite.)

If we relax the conditions slightly, the following construction gives examples of groups $G$ with subgroups $G_{n}$ for all natural numbers $n$, such that $G_{0}$ is finite 
(but $G_{n}$ infinite for $n>0$ ), and other examples where all $G_{n}$ are finite but the second condition is weakened to the pair of conditions

- $G_{n}^{\prime} \geq G_{n-1}$ for $n>0$,

- $G_{n}^{(n)}=G_{0}$.

Construction: Let $R$ be a ring with identity. (We are particularly interested in the case where $R$ is finite; but the construction works in general.) Let $I$ be the set of dyadic rational numbers in $[0,1]$, and $I_{n}$ the subset of $I$ in which the denominators are at most $2^{n}$. (So $I_{0}=\{0,1\}$.)

Our groups will be contained in the group of upper triangular matrices over $R$, where the index set of rows and columns is $I$. Let $e_{i j}$ be a symbol for each $i, j \in I$ with $i<j$. Our group will be generated by elements $x e_{i j}$ for all such $i, j$ and all $x \in R$; the relations are

- $x e_{i j} \cdot y e_{i j}=(x+y) e_{i j}$;

- for $i<j<k,\left[x e_{i j}, y e_{j k}\right]=x y e_{i k}$;

- if $j \neq k$ and $i \neq l$, then $\left[x e_{i j}, y e_{k l}\right]=1$.

Think of $x e_{i j}$ as the matrix $I+x E_{i j}$, where $E_{i j}$ has entry 1 in the $(i, j)$ position and 0 elsewhere.

Let $G_{n}$ be the subgroup generated by $x e_{i j}$ for $i, j \in I_{n}$, and $H_{d}$ be generated by $x_{i j}$ with $j-i \geq d$. The groups $G_{n}$ are isomorphic to the group of $\left(2^{n}+\right.$ 1) $\times\left(2^{n}+1\right)$ strictly upper triangular matrices over $R$, and so are finite if $R$ is finite. The groups $H_{d}$ are infinite if $d<1 ; G_{0}=H_{1}$ is isomorphic to the additive group of $R$.

Moreover, we see that

$$
\begin{aligned}
& G_{n}^{\prime}=G_{n} \cap H_{1 / 2^{n-1}} \geq G_{n-1} ; \\
& H_{d}^{\prime}=H_{2 d},
\end{aligned}
$$

with the convention that $H_{d}=\{1\}$ if $d>1$.

Hence the chains $\left(H_{1 / 2^{n}}\right)$ and $\left(G_{n}\right)$ of groups have the properties claimed.

\section{Infinite groups}

We have rather less to say about infinite groups.

The definition of integral applies equally to finite and infinite groups. Several of our results (Lemma 2.1, Proposition 3.1, Proposition 3.6(a) and (c), and Proposition 5.1) also apply to infinite groups. 
Following the first part of the proof of Theorem 2.2, we show:

Proposition 9.1: Let $G$ be finitely generated. If $G$ has an integral, then it has a finitely generated integral.

Proof. Suppose that $H$ is an integral of $G$. Take a finite generating set for $G$, and write each generator as a product of commutators of elements of $H$. Then the finite set of elements involved in these commutators generate a subgroup of $H$ whose derived group is $G$.

We mention a couple of classes of infinite groups which are integrable.

THEOREM 9.2: (a) Any abelian group is integrable.

(b) Any free group is integrable.

Proof. (a) We follow Guralnick's proof [5]: if $A$ is abelian, then the derived group of $A \nmid C_{2}$ is the subgroup $\left\{\left(a, a^{-1}\right): a \in A\right\}$ of the base group $A^{2}$ of the wreath product, which is clearly isomorphic to $A$.

(b) If $\alpha$ is an infinite cardinal, then $\left|F_{\alpha}\right|=\alpha$, so $\left|F_{\alpha}^{\prime}\right| \leq \alpha$. The inequality cannot be strict, since if $\left\{f_{i}: i \in \alpha\right\}$ is a free generating set for $F_{\alpha}$, then the elements $\left[f_{0}, f_{i}\right]$, for $i \in \alpha, i>0$, of the derived group are all distinct. So $F_{\alpha}^{\prime} \cong F_{\alpha}$.

For finite rank, we use the result of Nielsen [13], a special case of which asserts that the derived group of the free product $C_{m_{1}} * C_{m_{2}}$ is a free group of rank $\left(m_{1}-1\right)\left(m_{2}-1\right)$. So the derived group of $C_{2} * C_{n+1}$ is $F_{n}$.

REMARK 9.3: The free product of integrable groups may or may not be integrable. For example,

- $C_{2} * C_{2}$ is the infinite dihedral group, which is not integrable (by the same argument as for finite dihedral groups).

- $C_{3} * C_{3}$ is the derived group of $\operatorname{PGL}(2, \mathbb{Z})[6,16]$.

In view of part (a) above and our results on abelian groups, we could ask whether any abelian group $A$ has an integral $G$ with $|G: A|$ finite. For example, if $A=A^{2}$ (that is, every element of $G$ is a square), then the generalized dihedral group

$$
\left.G=\langle A, t\rangle \mid t^{2}=1, t^{-1} a t=a^{-1} \text { for all } a \in A\right\rangle
$$

is an integral of $A$ with $|G: A|=2$. But in general the answer is negative: 
Theorem 9.4: There exist infinite abelian groups $A$ having no integral $G$ with $|G: A|$ finite.

Proof. Following the arguments given in Section 4, we take $A$ to be the direct product of cyclic groups of orders $2^{k}$ for $k \in \mathbb{N}$.

Let $G$ be a 2-group such that $G^{\prime}=A$, and suppose for a contradiction that $|G / A|$ is finite. Then we can find subgroups $N \leq A$ with $N \unlhd G$ and $A / N$ finite and arbitrarily large. But $A / N=(G / N)^{\prime}$, contradicting Proposition 4.3.

In fact much more can be said about integrals of infinite abelian groups; this will be discussed in a later paper.

\section{Open problems}

We conclude with a list of problems arising from this study, some of which have already been mentioned.

Problem 10.1: Let $N$ be the set of positive integers $n$ for which every group of order $n$ is integrable (Theorem 7.1). Does $N$ have a density? What is its density? (We note that of the integers up to $10^{8}$, the number which lie in $N$ is 32261534.)

Problem 10.2: Find a good upper bound for the order of the smallest integral of an integrable group of order $n$.

Problem 10.3: True or false? For a fixed prime $p$, the proportion of groups of order $p^{n}$ which are integrable tends to 0 as $n \rightarrow \infty$.

Problem 10.4: (a) Let us call a group $G$ almost integrable if $G \times A$ is integrable for some abelian group $A$. We saw in Proposition 3.6(c) that, for centreless groups, "integrable" and "almost integrable" are equivalent. Which groups with non-trivial centre are almost integrable? (At the end of Section 3, we noted that $C_{2} \times D_{8}$ is integrable, so $D_{8}$ is almost integrable, but not integrable.)

(b) Does there exist a finite non-integrable group $G$, such that $G \times G$ is integrable? In particular, is $D_{8} \times D_{8}$ integrable? (As noted in Subsection 8.1 it has no integral of order less than 512.) 
Problem 10.5: For the three cases mentioned in Subsection 8.2 decide, for members $G$ of some class of subgroups of $U$, whether or not $G$ has an integral within $U$. In the context of the discussion following Lemma 6.2, especially Case 2 , we are particularly interested in the case where $U=\operatorname{Out}(T)$ for some simple group $T$ and $G$ is cyclic.

Problem 10.6: For which finite non-abelian groups $G$ is it true that, for all finite groups $H$ with $G^{\prime}=H^{\prime}$, it holds that $H$ is integrable if and only if $G$ is? (All finite abelian groups have this property, but it fails for $D_{8}$ and $Q_{8}$.)

Problem 10.7: One difficult problem is relating integrability of $G$ to that of $G / Z(G)$, or indeed $G / Z$ for any central subgroup $Z$ of $G$. Is there a cohomological tool that would help? One direction is trivial: if $G$ has an integral then so does $G / Z(G)$.

Let us suppose that $Z(G) \cong C_{p}$; put $H=G / Z(G)$. Then $G$ is "described" by a cohomology class $\gamma$ in the second cohomology group (or extension group) $H^{2}\left(H, C_{p}\right)$. Suppose that $H$ has an integral $K$. Does cohomology provide a tool to decide whether $\gamma$ is the restriction to $H$ of a class $\delta \in H^{2}\left(K, C_{p}\right)$ ? If so, then the corresponding extension is an integral of $G$.

Problem 10.8: Which integrable infinite groups $G$ have an integral $H$ such that the index of $G$ in $H$ is finite?

Problem 10.9: let $G$ be a locally finite group which is locally integrable (that is, every finite subset is contained in an integrable subgroup). Must $G$ be integrable? If so, must there be a locally finite integral?

Problem 10.10: Let $X$ be a $n$-set and $S_{n}$ the symmetric group on $X$. Does there exist an equivalence relation $\rho$ on $X$ such that the group $G \leq S_{n}$ of all permutations that preserve $\rho$ is integrable in $S_{n}$ ? We know this is false for the identity or universal relations; is it false for all equivalence relations?

Such a group $G$ is a direct product of wreath products $S_{a} \prec S_{b}$ of symmetric groups. As a preliminary step, we could ask whether a direct product of symmetric groups can be integrable.

Problem 10.11: Determine whether the following problem is undecidable: given a presentation $\langle X \mid R\rangle$ for a group $G$, is $G$ integrable? Are there decidable instances of this problem? For example, is the problem decidable for one-relator groups? 
Problem 10.12: It is known that the infinite finitely presented Thompson groups $T$ and $V$ are simple. On the other hand, Thompson's group $F$ has simple commutator subgroup, but is not itself simple. Is the group $F$ integrable?

Problem 10.13: (a) The class of all integrals of a given variety $V$ of groups is a variety of groups $W$. Given a base of identities for $V$, is it possible to find a base of identities for $W$ ?

(b) Let $G$ be a finite integrable group, and $W$ the variety of integrals of groups in $V=\operatorname{Var}(G)$. Is there an integral $H$ of $G$ such that $W=$ $\operatorname{Var}(H)$ ?

Related to the previous problem we have the following.

Problem 10.14: Is it possible to classify the finite sets $A \subseteq F_{2}$, the 2-generated free group, such that the group

$$
\langle a, b \mid w(a, b)=1=w(b, a)(w \in A)\rangle
$$

satisfies $w(x, y)=1$, for all $w \in A$ ?

Problem 10.15: Every group in a variety of abelian groups has integral. Are there other varieties with this property?

Problem 10.16: For any integrable group there is a smallest integral; how many different integrals of smallest order can there be?

Problem 10.17: Is it true that no Coxeter group with connected diagram, apart from $C_{2}$, is integrable?

Problem 10.18: Produce some algorithms and effective GAP code to find integrals of a given group.

Problem 10.19: As there is a classification of the groups in which all subgroups are normal, classify the groups in which all subgroups are integrable.

Problem 10.20: It makes sense to adapt the integrability concept to Lie algebras via the derived subalgebra. Is it true that a Lie algebra is integrable if and only if the corresponding Lie group is?

PROBLem 10.21: Is there a ring theoretic analogue of the results in this paper, now taking $[a, b]=a b-b a$ ? 
Observe that in general, the commutators of all pairs of elements in a ring form a subring, but they do not necessarily form an ideal [9]. Therefore the ring theory literature considers the commutator as the ideal generated by all pairs $[a, b]$. Nevertheless, they form a right ideal if and only if they form a left ideal: $(a b-b a) c=a(b c-c b)+(a c) b-b(a c)$.

\section{Acknowledgments}

The first, second and fourth authors gratefully acknowledge the support of the Fundação para a Ciência e a Tecnologia (CEMAT-Ciências FCT project UID/Multi/04621/2013).

The third and fourth authors are members of the Gruppo Nazionale per le Strutture Algebriche, Geometriche e le loro Applicazioni (GNSAGA) of the Istituto Nazionale di Alta Matematica (INdAM). The fourth author gratefully acknowledges the support of the Fundação de Amparo à Pesquisa do Estado de São Paulo (FAPESP Jovens Pesquisadores em Centros Emergentes grant 2016/12196-5), of the Conselho Nacional de Desenvolvimento Científico e Tecnológico (CNPq Bolsa de Produtividade em Pesquisa PQ-2 grant 306614/2016$2)$.

\section{References}

[1] A. Abdollahi, Integral of a group, in Proceedings of 29th Iranian International Conference on Mathematics, Amirkabir University of Technology, Iran, 1998.

[2] K. Filom and B. Miraftab, Integral of groups, Comm. Algebra, 45 (2017), 1105-1113.

[3] D. Gorenstein, Finite Groups, Second edition. Chelsea Publishing Co., New York, 1980. xvii+519 pp.

[4] Group Properties, Not all groups are commutator-realizable, http://groupprops .subwiki.org/wiki/Derived_subgroup\#Group_properties

[5] R. M. Guralnick, On groups with decomposable commutator subgroups. Glasgow Math. J. 19 (1978), no. 2, 159-162.

[6] G. A. Jones and John S. Thornton, Automorphisms and congruence subgroups of the extended modular group, J. London Math. Soc. (2) 34 (1986), 26-40.

[7] MathOverflow, Finite nonabelian groups of odd order, https://mathoverflow.net/questions/31553/finite-nonabelian-groups-of-odd-order/ 31558\#31558

[8] MathOverflow, Realizing groups as commutator subgroups, https://mathoverflow.net/questions/85540/realizing-groups-as-commutator-subgroups

[9] J.B. McLeod, On the commutator subring. Quart. J. Math. Oxford Ser. (2) 9 (1958), 207-209. 
[10] M. D. Miller, Existence of finite groups with classical commutator subgroup. J. Austral. Math. Soc. Ser. A 25 (1978), no. 1, 41-44.

[11] B. H. Neumann, Ascending derived series, Compositio Math. 13 (1956), 47-64.

[12] M. F. Newman, On a class of nilpotent groups, Proceedings of the London Mathematical Society, Third Series, 10 (1960), 365-375.

[13] J. Nielsen, Kommutatorgruppen for det frie produkt af cykliske grupper, Matematisk Tidsskrift B (1948), 49-56.

[14] D. Robinson, A Course in the Theory of Groups, Springer, New York, 1995.

[15] J. J. Rotman, Introduction to the Theory of Groups, Springer, New York, 1995.

[16] R. Sahin, O. Bizim and I. N. Cangul, Commutator subgroups of the extended Hecke groups $\bar{H}\left(\lambda_{q}\right)$, Czechoslovak Math. J. 54 (2004), 253-259.

[17] H. Wielandt, Eine Verallgemeinerung der invarianten Untergruppen, Math. Z. 45 (1939), 209-244.

[18] H. Zassenhaus, Lehrbuch der Gruppentheorie, 1. Band, Teubner, Leipzig \& Berlin, 1937. 